\title{
Prospective analysis of the association between skeletal-related events and quality of life in patients with advanced lung cancer (CSP-HOR13)
}

\author{
HIROSHI KUNIKANE ${ }^{1}$, ISAO YOKOTA ${ }^{2}$, NOBUYUKI KATAKAMI ${ }^{3}$, KOJI TAKEDA $^{4}$, \\ KOICHI TAKAYAMA ${ }^{5}$, TOSHIYUKI SAWA ${ }^{6}$, HIROSHI SAITO ${ }^{7}$, MASAO HARADA $^{8}$, SOICHIRO YOKOTA ${ }^{9}$, \\ KIYOSHI ANDO ${ }^{10}$, YUKO SAITO ${ }^{11}$, YASUO OHASHI ${ }^{12}$ and KENJI EGUCHI ${ }^{13}$
}

\begin{abstract}
${ }^{1}$ Department of Palliative Medicine, Yokohama Municipal Citizen's Hospital, Yokohama, Kanagawa 240-8555;
${ }^{2}$ Department of Biostatistics, Kyoto Prefectural University of Medicine, Kyoto, Kyoto 602-8566; ${ }^{3}$ Division of Integrated

Oncology, Institute of Biomedical Research and Innovation, Kobe, Hyōgo 650-0047; ${ }^{4}$ Division of Medical Oncology, Osaka City General Hospital, Osaka, Osaka 534-0021; ${ }^{5}$ Department of Pulmonary Medicine, Kyoto Prefectural University of

Medicine, Kyoto, Kyoto 602-8566; ${ }^{6}$ Division of Respiratory Medicine and Oncology, Gifu Municipal Hospital, Gifu,

Gifu 500-8513; ${ }^{7}$ Department of Respiratory Medicine, Aichi Cancer Center Aichi Hospital, Okazaki, Aichi 444-0011;

${ }^{8}$ Department of Respiratory Medicine, National Hospital Organization Hokkaido Cancer Center, Sapporo, Hokkaido 003-0804;

${ }^{9}$ Department of Respiratory Medicine, Toneyama National Hospital, Toyonaka, Osaka 560-0045;

${ }^{10}$ Division of Hematology/Oncology, Tokai University School of Medicine, Isehara, Kanagawa 259-1193;

${ }^{11}$ Clinical Trial Coordination Office, Shizuoka Cancer Center, Shizuoka, Shizuoka 411-8777;

${ }^{12}$ Department of Integrated Science and Engineering for Sustainable Society, Chuo University, Tokyo 112-8551; ${ }^{13}$ Medical Oncology, Teikyo University School of Medicine, Tokyo 173-8606, Japan
\end{abstract}

Received November 20, 2017; Accepted October 15, 2018

DOI: $10.3892 / \mathrm{ol} .2018 .9680$

\begin{abstract}
A prospective study has previously reported on the incidence of bone metastasis (BM) and skeletal-related events (SREs) in patients with advanced lung cancer. The aim of the present study was to prospectively investigate how the quality of life (QOL) of patients with advanced lung cancer was affected by SREs. Patients with stage IIIB or IV non-small cell lung cancer (NSCLC) or small cell lung cancer (SCLC) at any stage were followed up every four weeks to determine if they had developed SREs. QOL questionnaires were conducted at enrollment, at 3- and 12-months later and at 1 month after the onset of SREs, using QOL scores including the EuroQOL-5 Dimension (EQ-5D), Functional Assessment of Cancer Therapy-General (FACT-G) and activities of daily living (ADL) scores obtained by the Barthel Index. A total of 274 patients were enrolled in the study. At enrollment the
\end{abstract}

Correspondence to: Dr Hiroshi Kunikane, Department of Palliative Medicine, Yokohama Municipal Citizen's Hospital, 56 Okazawa-cho, Hogogaya-ku, Yokohama, Kanagawa 240-8555, Japan

E-mail: hi00-kunikane@city.yokohama.jp

Key words: lung cancer, skeletal-related event, quality of life, EuroQOL-5 dimension, functional assessment of cancer therapy-general, barthel index
EQ-5D and Barthel Index scores were lower in patients with SREs compared with patients without SREs. A chronological analysis revealed no statistically significant changes in either QOL or ADL in any of the patients. For 14 patients in whom QOL data was collected following the onset of SREs, the evaluation undertaken on the four subscales of the FACT-G revealed a significant decline in emotional functioning following the onset of SREs.

\section{Introduction}

Lung cancer is one of the most common malignant neoplasms worldwide. The majority of patients diagnosed with lung cancer already have distant metastases, and are diagnosed as in an advanced-stage disease (1). The skeleton is one of the most frequent metastatic sites in patients with advanced lung cancer $(2,3)$, and the incidence of bone metastasis (BM) is approximately $30-40 \%$, when diagnosed in a clinical course of lung cancer.

Cancer patients with BM should prevent skeletal complications that increase suffer and may require high medical costs (4). Such complications are usually indicated as skeletal related events (SREs). However, incidence of SREs in patients with lung cancer has varied across several reports that investigated their clinical courses (5-7). While SREs are also thought to exert a negative impact on quality of life (QOL) in patients with lung cancer, few reports regarding the association between SREs and QOL have been published (8-10). 
In the previous work, we reported the incidence of BM and SREs in patients with advanced lung cancer, as a prospective study (11). The aim of the current study was to prospectively investigate how QOL was affected by SRE in patients with advanced lung cancer, as well as incidence of BM and SRE.

\section{Materials and methods}

Patient enrollment and study design. This study was a prospective multicenter cohort study. The eligibility criteria included newly-diagnosed patients with stage IIIB or IV non-small cell lung cancer (NSCLC) or small cell lung cancer (SCLC) in any stage, whose ages were over 20-years old, and who had provided written informed consent. All patients were required to have not received chemotherapy or bisphosphonate therapy.

Treatment for lung cancer and the administration of zoledronate were at the discretion of the investigator, for the enrolled patients. Denosumab was not approved during the study period in Japan. The present study was approved by the institutional review boards of the respective institutions, and was conducted in compliance with international guidelines regulating patient safety.

Evaluation of BM, SREs, and date collection. The physicians and clinical research coordinators (CRCs) collected data every four weeks during the six month periods following enrollment, and every three months thereafter. The data included Eastern Cooperative Oncology Group Performance Status (ECOG-PS), body weight, blood sampling (to check hypercalcemia, bone, or other metastases), use of zoledronate or analgesics, and pain scale. We checked chest CT, bone scintigraphy, and roentgenograms of the thoracic and lumbar bones at the time of study enrollment. A chest CT was performed every three months and bone scintigraphy was performed every six months. Treatment for lung cancer and use of zoledronate were undertaken at the discretion of the investigators. When bone metastases were suspected, the patient underwent a CT to determine bone conditions or an MRI and X-ray for the diagnosed BM sites.

QOL assessment and definition of SREs. QOL was assessed via the EuroQOL-5 Dimension (EQ-5D) (12) and Functional Assessment of Cancer Therapy-General (FACT-G) (13). ADL was evaluated via the Barthel Index (14). These QOL questionnaires were conducted at the time of enrollment, at both three- and twelve-months later, and also one month after the onset of SREs. SREs are defined as pathologic fracture, radiation or surgery to bone lesion, spinal cord compression, or hypercalcemia. Each QOL questionnaire was analyzed only in patients from whom all answers were collected.

Statistical analysis. The sample size has been described in the previous report (11). The target number of cases was set at 50, as a number of cases for which descriptive statistics of some accuracy can be calculated, even for patients with stage IIIB NSCLC, which is anticipated to have comparatively small enrollment, for a total of roughly 400 cases from the percentage of each cancer type. The sample size was determined to answer another research question about the incidence of SREs, and the present analysis was a secondary use of those data.
Table I. Patients characteristics at enrollment $(n=274)$.

\begin{tabular}{|c|c|}
\hline Characteristics & Total \\
\hline \multicolumn{2}{|l|}{ Sex } \\
\hline Male & 193 \\
\hline Female & 81 \\
\hline Median age (range) & $68(35-89)$ \\
\hline \multicolumn{2}{|l|}{ Histology } \\
\hline NSCLC & 197 \\
\hline Stage IIIB & 73 \\
\hline Stage IV & 124 \\
\hline SCLC & 77 \\
\hline LD & 30 \\
\hline ED & 47 \\
\hline \multicolumn{2}{|l|}{ ECOG-PS } \\
\hline 0 & 76 \\
\hline 1 & 171 \\
\hline 2 & 23 \\
\hline 3 or 4 & 4 \\
\hline \multicolumn{2}{|l|}{ BM } \\
\hline+ & 78 \\
\hline- & 196 \\
\hline \multicolumn{2}{|l|}{ SRE } \\
\hline+ & 24 \\
\hline- & 250 \\
\hline
\end{tabular}

Background characteristics and types of SREs are summarized by frequency and proportion. The mean values of the EQ-5D, FACT-G, and Barthel index, among the baseline BM and SRE status types, were drawn by box-whisker plot and compared by one-way analysis of variance. In the patients who had SRE for the first time within the follow-up period, the changes in EQ-5D, FACT-G, and Barthel index by SRE occurrence were compared by paired t-test. In addition, the changes in the subscales of the FACT-G were evaluated in the same manner. P-value for comparisons between SRE +/- and between $\mathrm{BM}+/$ - were calculated concurrently using contrasts. At two-sided $\mathrm{P}<0.05$ was considered to indicate a statistically significant difference. All statistical analyses were performed using SAS v9.2 (SAS Institute, Cary, NC, USA).

\section{Results}

Patients backgrounds at enrollment. A total of 274 patients were enrolled in this study from April 2007 to December 2009. The patients characteristics are summarized in Table I. Patient ages ranged from 35 to 89 years (median age, 68 years), and male/female $=193 / 81$, ECOG-PS0/1/2/3=76/171/23/4, BM+/-=78/196, SREs+/-=24/250, SCLC/NSCLC $=77 / 197$, and Stage IIIB/IV=73/124. 


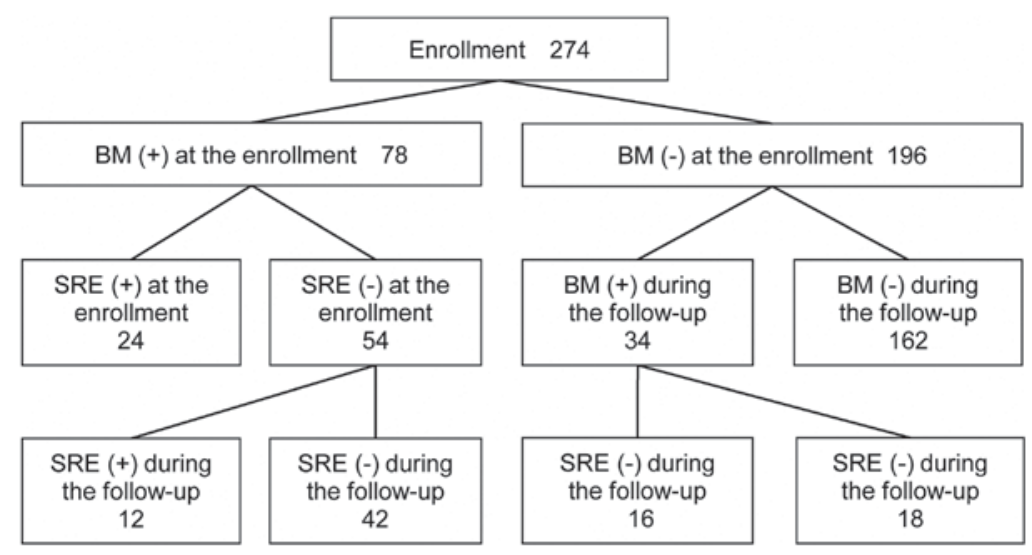

Figure 1. Numbers of BM and SREs at enrollment and during the follow-up periods. BM, bone metastasis; SREs, skeletal-related events.

Incidence of BM and SREs. BM and SREs both at enrollment and during the follow-up periods are summarized in Fig. 1. As previously reported (11), the median follow-up period was 13.8 months (0-28.5 month). A total of 78 patients $(28 \%$ of all enrolled patients and $62 \%$ of stage IV patients) already had $\mathrm{BM}$ at enrollment. Among these patients, 24 had accompanying SREs, while an additional 12 developed SREs during the follow up. Among the 196 patients without initial BM, 34 developed BM. Among these 34 patients, 16 developed SREs during the follow-up.

The types of SREs at enrollment were: Pathologic fracture in nine patients; radiation to bone lesion in 22; spinal cord compression in two; and hypercalcemia of malignancy in one. The types of new SREs during the follow-up period were: Pathologic fracture in five patients; radiation to bone lesion in 23; spinal cord compression in one; and hypercalcemia of malignancy in five.

QOL of the patients. The EQ-5D, FACT-G, and Barthel Index scores at enrollment were summarized according to three patient groups classified by BM and SRE, and are respectively shown in Fig. 2A-C. QOL data were obtained from nearly all patients at the enrollment. Among patients with BM at enrollment, those who already had SREs had lower EQ-5D (mean=0.7) and Barthel Index (mean=94.8) scores than those without SREs (mean of EQ-5D=0.8, mean of Barthel Index=98.9), and the differences were statistically significant ( $\mathrm{P}=0.013$ for $\mathrm{EQ}-5 \mathrm{D}, \mathrm{P}=0.006$ for Barthel Index). Such significant lower scores in patients who already had SREs were observed when compared to either those who did not have BM or those had BM but no SREs. No significant difference was observed for FACT-G between patients with $(m e a n=66.8)$ and without (mean=71.6) SREs at enrollment $(\mathrm{P}=0.225)$. In addition, none of the EQ-5D, FACT-G, or Barthel Index scores showed a significant difference between patients who did not have BM and those had BM but no SREs.

Changes in QOL and ADL in the patients during the study period are summarized in Fig. 3A-C. A chronological analysis showed no statistically significant change in the EQ-5D, FACT-G, or Barthel Index scores of all patients for whom the QOL or ADL evaluation was performed.

QOL data were collected for 14 (EQ-5D) or 13 (FACT-G and Barthel Index) patients out of 28 who had SREs during the

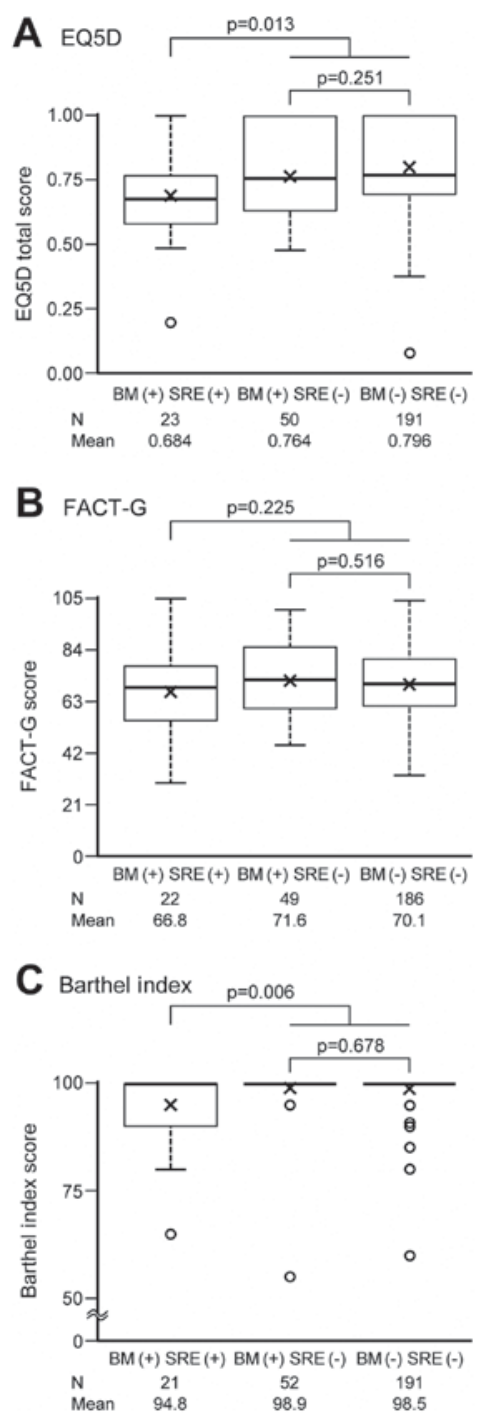

Figure 2. (A) EQ-5D, (B) FACT-G and (C) Barthel Index scores at enrollment, according to three patient groups classified by BM and SREs using one-way analysis of variance. P-value for comparisons between SRE +/- and between $\mathrm{BM}+/$ - were calculated from post-hoc orthogonal contrast test of $(2,-1,-1)$ and $(0,1,-1)$ in one-way analysis of variance. Multiplicity was not adjusted because of exploratory analysis. Thick horizontal lines delineate median values, while the box boundaries represent the 25 th and 75 th percentiles. ' $x$ ' represents mean values. Whiskers outside the boxes delineate the maximum or minimum of 1.5 times the interquartile range. BM, bone metastasis; EQ-5D, EuroQOL-5 Dimension; FACT-G, Functional Assessment of Cancer Therapy-General; SREs, skeletal-related events. 

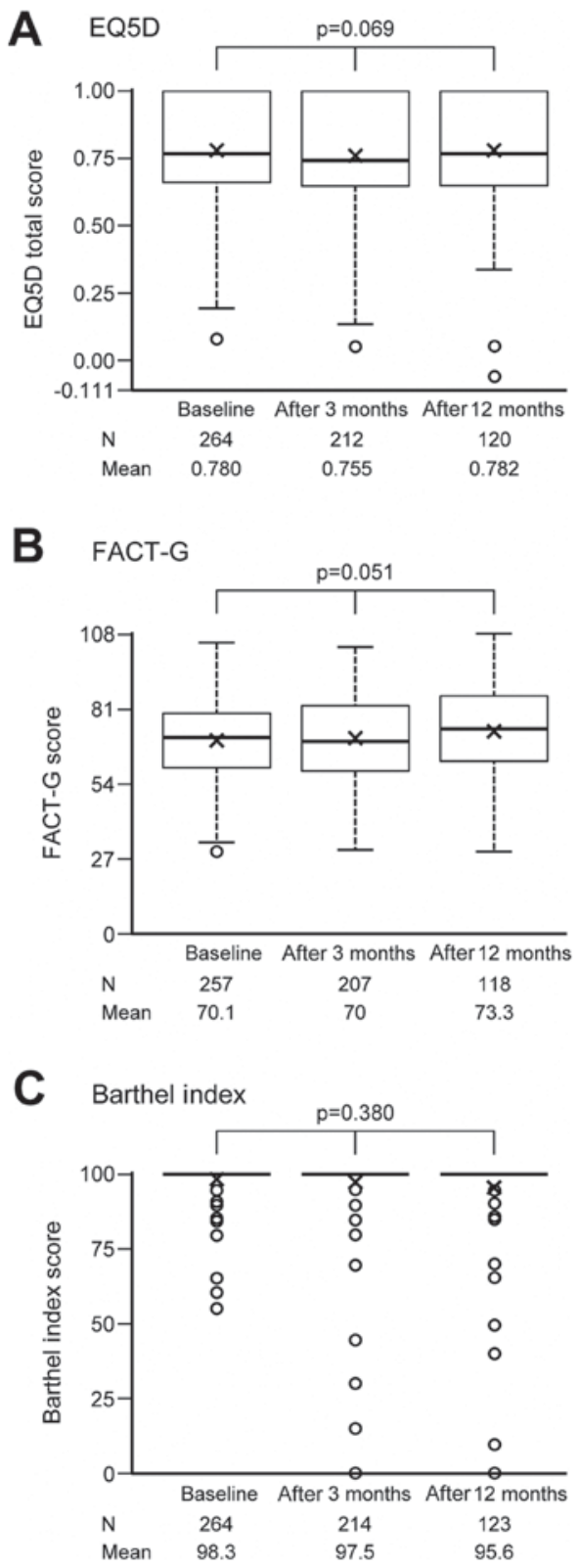

Figure 3. Changes in the (A) EQ-5D, (B) FACT-G and (C) Barthel index of all patients, by using one-way analysis of variance with subject-specific random intercept. Thick horizontal lines delineate median values, while the box boundaries represent the 25 th and 75 th percentiles. ' $x$ ' represents mean values. Whiskers outside the boxes delineate the maximum or minimum of 1.5 times the interquartile range. EQ-5D, EuroQOL-5 Dimension; FACT-G, Functional Assessment of Cancer Therapy-General.

follow-up. Changes in QOL after the SREs are summarized in Fig. 4A-C. For those 13 or 14 patients, QOL and ADL scores changed by -0.05 in EQ-5D, by -8.5 in FACT-G, and by -4.6 in Barthel Index. However, none of these decreases were statistically significant.

Changes in the QOL by the FACT-G after the onset of SREs, for each functioning subscale are summarized in Fig. 5. An analysis of FACT-G by its four subscales (physical, social/family, emotional, and functional) showed that the emotional subscale decreased by 3.77 (95\%CI: 6.83, 0.71), which was statistically significant $(\mathrm{P}=0.02)$. However, physical, social/family, and functional subscales showed no significant changes.
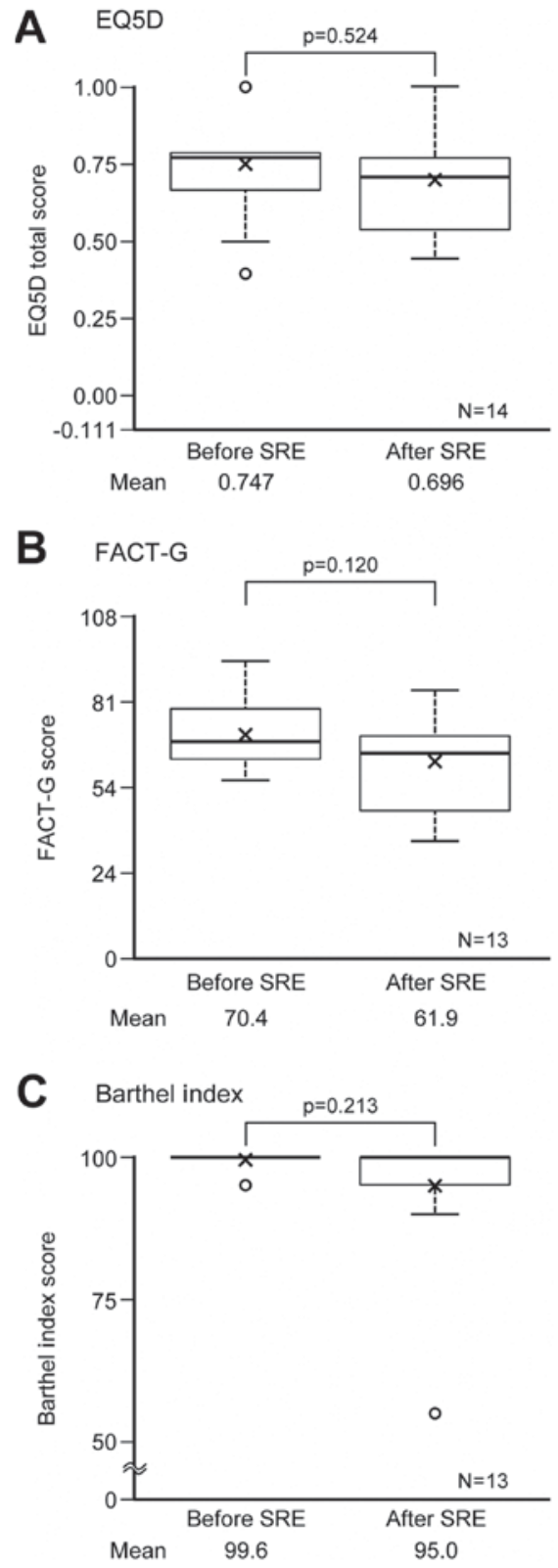

Figure 4. Changes in the (A) EQ-5D, (B) FACT-G and (C) Barthel index after the onset of SREs by using paired t-test. Thick horizontal lines delineate median values, while the box boundaries represent the 25th and 75 th percentiles. ' $x$ ' represents mean values. Whiskers outside the boxes delineate the maximum or minimum of 1.5 times the interquartile range. EQ-5D, EuroQOL-5 Dimension; FACT-G, Functional Assessment of Cancer Therapy-General; SREs, skeletal-related events.

\section{Discussion}

This is another analysis which focuses on the QOL of the patients with lung cancer, in whom the incidence of BM and SREs were prospectively investigated. In the present study, QOL data of the patients were also prospectively collected, according to the methods. As shown in the results, we reported their incidence at enrollment, as well as those occurred subsequently during the follow-up.

The association between SRE and QOL has frequently been studied from the point of an administration of zoledronate. While a number of reports have shown that its administration resulted in a decline of SRE, the accompanying effect 


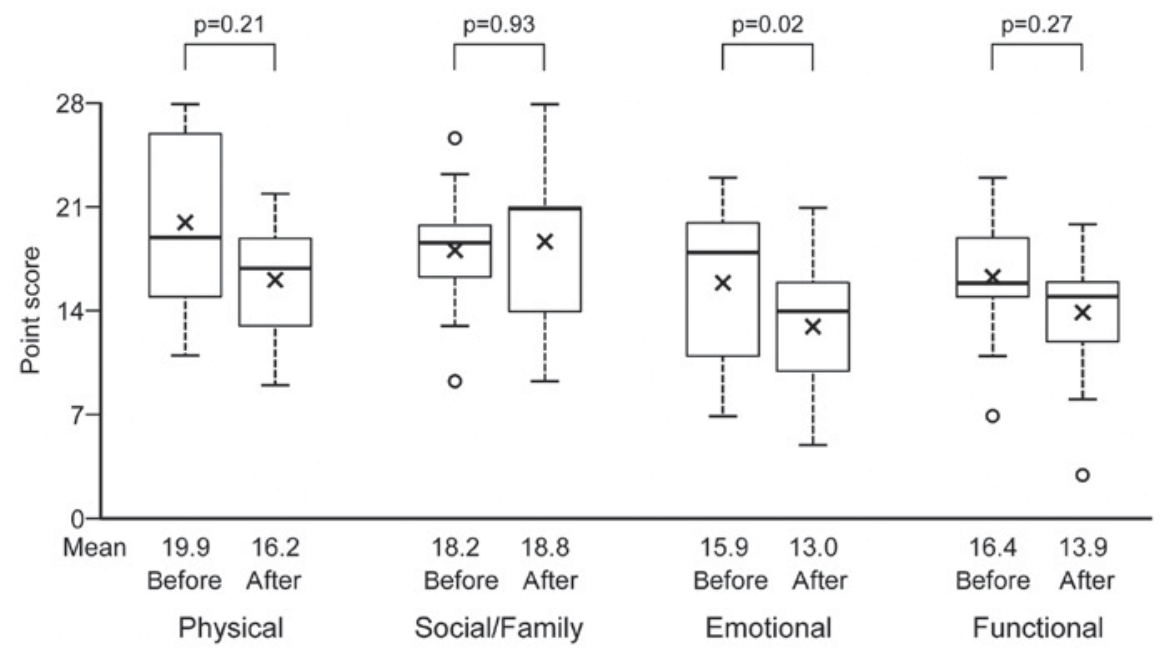

Figure 5. Changes in QOL by the FACT-G after the onset of SREs for each functioning subscale, by using paired t-test. Thick horizontal lines delineate median values, while the box boundaries represent the 25th and 75th percentiles. ' $x$ ' represents mean values. Whiskers outside the boxes delineate the maximum or minimum of 1.5 times the interquartile range. FACT-G, Functional Assessment of Cancer Therapy-General; QOL, quality of life; SREs, skeletal-related events.

on QOL remains controversial $(8-10,15,16)$. In all three of the studies that reported a positive effect of zoledronate on QOL in patients with cancer, the number of patients was rather small and the studies were retrospective $(6,17,18)$. To our knowledge, the present study is the first that prospectively investigated the QOL of the patients, by scheduled evaluation, including after the occurrence of SRE.

Several previous studies have been planned to observe QOL. However, the analyses were performed on a limited population of patients, such as those who were undergoing adjuvant chemotherapy (19), in the advanced or recurrent stage $(20,21)$, or who were undergoing maintenance chemotherapy (22). None of those studies reported a definite decline of QOL, and the present study showed a similar result. With regard to QOL during the one year after study enrollment, a definite change in QOL was not observed, in spite of a slight decline indicated by an evaluation with the Barthel index.

In the present study, the effect of SREs on QOL was analyzed using two methods. First, QOL was compared at study enrollment, between patients who already had and did not have SREs, and the patients with SREs showed worse QOL or ADL scores. This result suggests that SREs had a negative impact on QOL and ADL in the patients, for whom various types of supportive care was not sufficiently given.

In addition, the change in QOL and ADL was analyzed by comparing values before and after the onset of SREs, however, a significant decline was not observed after SRE onset. This result may be due to the small number of cases in which an evaluation of QOL was performed, and may also be due to the evaluation in patients with some limited types of SREs. All of the fourteen patients, in whom QOL was evaluated, had either radiation therapy to skeletal lesion or hypercalcemia as SREs, which could be relatively manageable to keep good QOL. On the other hand, QOL could be evaluated in none of five patients, who had a pathologic fracture after the enrollment and might cause functional impairment.

There was a tendency for QOL or ADL to decline in the fourteen evaluated patients. This result may suggest that SREs exerted a more serious impact on QOL and ADL in the patients as a whole, as it is possible that those who did not receive evaluations had worse QOL or ADL compared to those for whom an evaluation was performed due to the maintenance of relatively better conditions.

One notable finding in the present study is that the emotional score was declined after the onset of SREs, even though the total FACT-G scores did not indicate a significant decline in QOL. Although SREs would appear to be more associated with physical function, the results did not match such expectations. It is possible that the significant decline in FACT-G emotional score could be due to the multiplicity of comparisons.

The present study has some limitations, the most notable of which is that QOL could be evaluated in only fourteen cases after the onset of SREs, although 28 patients experienced various types of SREs. We could not evaluate nearly half of patients with SREs after the enrollment. As previously mentioned, this lack of QOL assignment might under-evaluate the decline in QOL after the onset of SREs, by only evaluating patients in good health and excluding patients who might have had more serious impairments and could not have a QOL evaluation. It is possible that the 14 patients did not represent the whole population who newly had SREs after the enrollment. Some patients might have multiple SREs, but we could not analyze the association between QOL and each SRE, although the association may differ according to type of SREs.

Such elimination might also result in under-evaluation in the chronological analyses of QOL and ADL at enrollment, and at three- and twelve-months later. It is possible that a non-significant change in QOL or ADL was due to some patients having serious impairments, so that they could not have assignments.

Another limitation is that the selected population of patients who were predominantly enrolled in this study, already had the support of a palliative care team, including a clinical research coordinator (CRC), as the enrollment was not consecutive. It is possible that such support by various 
professionals resulted in the maintenance of relatively good QOL even after the onset of SREs, so that this population did not represent the whole population of patients with lung cancer, although we do not have concrete data of supportive care which the patients had taken during the study period.

Finally, the present analysis was performed only in Japanese population and could not be enough to discuss about international relevancy.

In Conclusion, QOL and ADL in the patients with advanced lung cancer was negatively affected by SREs just after the diagnosis, when measured by the EQ-5D and the Barthel Index. Although it was not proven that their QOL and ADL was affected by the onset of SREs, when FACT-G was evaluated by the four factors, emotional functioning significantly declined after SREs.

\section{Acknowledgements}

Not applicable.

\section{Funding}

Supported by Health Outcomes Research (CSP-HOR) of the Public Health Research Foundation.

\section{Availability of data and materials}

The datasets used and/or analyzed during the present study are available from the corresponding author on reasonable request.

\section{Authors' contributions}

YO and KE conceived the study. HK, YS, YO, and KE designed the study. HK, NK, KojT, KoiT, TS, HS, MH, SY, and KA collected data. IY analyzed data. HK, IY, and NK wrote the manuscript. All authors reviewed the manuscript and approved the final version of the manuscript.

\section{Ethics approval and consent to participate}

This study was approved by the institutional review boards of the respective institutions (Yokohama Municipal Citizen's Hospital, Institute of Biomedical Research and Innovation, Osaka City General Hospital, Kyoto Prefectural University of Medicine, Gifu Municipal Hospital, Aichi Cancer Center Aichi Hospital, National Hospital Organization Hokkaido Cancer Center, and Toneyama National Hospital) and was conducted in compliance with international guidelines regulating patient safety.

\section{Patient consent for publication}

Patients provided written informed consent for data collection and analysis, and publication.

\section{Competing interests}

The authors declare that they have no competing interests.

\section{References}

1. Stinchcombe TE, Lee CB and Socinski MA: Current aproaches to advanced-stage non-small-cel lung cancer: First-line therapy in patients with a good functional status. Clin Lung Cancer 7 (Suppl 4): S111-S117, 2006.

2. Coleman RE: Clinical features of metastatic bone disease and risk of skeletal morbidity. Clin Cancer Res 12: 6243s-6249s, 2006.

3. Sugiura H, Yamada K, Sugiura T, Hida T and Mitsudomi T: Predictors of survival in patients with bone metastasis of lung cancer. Clin Orthop Relat Res 466: 729-736, 2008.

4. Botteman M, Barghout V, Stephens J, Hay J, Brandman J and Aapro M: Cost effectiveness of bisphosphonates in the management of breast cancer patients with bone metastases. Ann Oncol 17: 1072-1082, 2006.

5. Tsuya A, Kurata T, Tamura K and Fukuoka M: Skeletal metastases in non-small cell lung cancer: A retrospective study. Lung Cancer 57: 229-232, 2007.

6. Rosen LS, Gordon D, Tchekmedyian S, Yanagihara R, Hirsh V, Krzakowski M, Pawlicki M, de Souza P, Zheng M, Urbanowitz G, et al: Zoledronic acid versus placebo in the treatment of skeletal metastases in patients with lung cancer and other solid tumors: A phase III, double blind, randomized trial-the zoledoronic acid lung cancer and other solid tumors study group. J Clin Oncol 21: 3150-3157, 2003.

7. Rosen LS, Gordon D, Tchekmedyian NS, Yanagihara R, Hirsh V, Krzakowski M, Pawlicki M, De Souza P, Zheng M, Urbanowitz G, et al: Long-term efficacy and safety of zoledronic acid in the treatment of skeletal metastases in patients with nonsmall cell lung carcinoma and other solid tumors: A randomized, phase III, double blind, placebo-controlled trial. Cancer 100: 2613-2221, 2004.

8. Ishiwata T, Hirose T, Hirama M, Miura K, Iwakami S, Tominaga S, Adachi $\mathrm{M}$ and Takahashi K: A feasibility study of zoledronic acid combined with carboplatin/nedaplatin plus paclitaxel in patients with non-small cell lung cancer with bone metastases. Tumori 97: 568-572, 2011.

9. Addeo R, Nocera V, Faiola V, Vincenzi B, Ferraro G, Montella L, Guarrasi R, Rossi E, Cennamo G, Tonini G, et al: Management of pain in elderly patients receiving infusion of zoledronic acid for bone metastasis: A single-institution report. Support Care Cancer 16: 209-214, 2008.

10. Facchini G, Caraglier M, Santini D, Nasti G, Ottaiano A, Striano S, Maiolino P, Ruberto M, Fiore F, Tonini G, et al: The clinical response on bone metastasis from breast and lung cancer during treatment with zoledronic acid is inversely correlated to skeletal related events (SRE). J Exp Clin Cancer Res 26: 307-312, 2007.

11. Katakami N, Kunikane H, Takeda K, Takayama K, Sawa T, Saito H, Harada M, Yokota S, Ando K, Saito Y, et al: Prospective study on the incidence of bone metastasis (BM) and skeletal-related events (SREs) in patients (pts) with stage IIIB and IV lung cancer-CSP-HOR 13. J Thorac Oncol 9: 231-238, 2014.

12. Tsuchiya A, Ikeda S, Ikegami N, Nishimura S, Sakai I, Fukuda T, Hamashima C, Hisashige A and Tamura M: Estimating an EQ-5D population value set: The case of Japan. Health Econ 11: 341-353, 2002.

13. Cella DF, Bonomi AE, Lloyd SR, Tulsky DS, Kaplan E and Bonomi P: Reliability and validity of the functional assessment of cancer therapy-lung (FACT-L) quality of life instrument. Lung Cancer 12: 199-220, 1995.

14. Mahoney FI and Barthel DW: Functional evaluation; the Barthel Index. Md State Med J 14: 61-65, 1965.

15. Kretzschmar A, Wiegel T, AI-Batran SE, Hinrichs HF, Kindler M, Steck T, Illiger HJ, Heinemann V, Schmidt K, Haus U, et al: Rapid and sustained influence of intravenous zoledronic acid on course of pain and analgesics consumption in patients with cancer with bone metastases: A multicenter open-label study over 1 year. Support Cancer Ther 4: 203-210, 2007.

16. Mystakidou K, Katsouda E, Parpa E, Kouskouni E, Chondros C, Tsiatas ML, Galanos A and Vlahos L: A prospective randomized controlled clinical trial of zoledronic acid for bone metastases. Am J Hosp Palliat Care 23: 41-50, 2006.

17. Chow E and Bottomley A: Understanding the EORTC QLQ-BM22, the module for patients with bone metastases. Expert Rev Pharmacoecon Outcomes Res 9: 461-465, 2009. 
18. Koizumi M, Yoshimoto M, Kasumi F, Iwase T and Ogata E Post-operative breast cancer patients diagnosed with skeletal metastasis without bone pain had fewer skeletal-related events and deaths than these with bone pain. BMC Cancer 10: 423, 2010.

19. Bezjak A, Lee CW, Ding K, Brundage M, Winton T, Graham B, Whitehead M, Johnson DH, Livingston RB, Seymour L and Shepherd FA: Quality-of-life outcomes for adjuvant chemotherapy in early-stage non-small-cell lung cancer: Result from a randomized trial, JBR. 10. J Clin Oncol 26: 5052-5059, 2008

20. Mills M, Murray LJ, Johnston BT, Cardwell C and Donnelly M: Does a patient-held quality-of-life diary benefit patients with inoperable lung cancer? J Clin Oncol 27: 70-77, 2009.
21. Efficace F, Bottomley A, Smit EF, Lianes P, Legrand C Debruyne C, Schramel F, Smit HJ, Gaafar R, Biesma B, et al: Is a patient's self-reported health-related quality of life a prognostic factor for survival in non-small-cell lung cancer patients? A multivariate analysis of prognostic factors of EORTC study 08975. Ann Oncol 17: 1698-1704, 2006.

22. Belani CP, Brodowicz T, Ciuleanu TE, Krzakowski M, Yang SH, Franke F, Cucevic B, Madhavan J, Santoro A, Ramlau R, et al: Quality of life in patients with advanced non-small-cell lung cancer given maintenance treatment with pemetrexed versus placebo (H3H-MC-JMEN): Result from a randomised, double-blind, phase 3 study. Lancet Oncol 13: 292-299, 2012. 